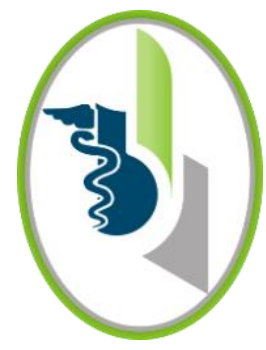

ACCESO ABIERTO

Para citaciones: Sáenz, J., Tatis, K. Bohórquez, J., Guzmán, R. (2021). Aspectos fisiopatológicos y

manifestaciones neurológicas asociadas a la infección por SARS-CoV-2/COVID-

19. Revista Ciencias Biomédicas, 10(1), 39-54.

Recibido: 12 de noviembre de 2020 Aprobado: 10 de enero de 2021

Autor de correspondencia: José de Jesús Bohórquez-Rivero josejbohorquez@gmail.com

Editor: Inés Benedetti. Universidad de Cartagena-Colombia.

Copyright: (C) 2021. Sáenz, J., Tatis, K., Bohórquez, J., Guzmán, R. Este es un artículo de acceso abierto, distribuido bajo los términos de la licencia https://creativecommons.org/licenses/by-ncsa/4.0/ la cual permite el uso sin restricciones, distribución y reproducción en cualquier medio, siempre y cuando el original, el autor y la fuente sean acreditados.

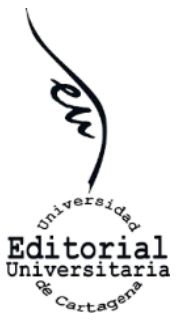

\section{Aspectos fisiopatológicos y manifestaciones neurológicas asociadas a la infección por SARS- CoV-2/COVID-19}

\author{
Pathophysiologic aspects and neurological manifestations \\ associated with SARS-CoV-2 infection/COVID-19
}

\author{
José David Sáenz-López ${ }^{1 D}$, Keyner Enrique Tatis-Villamizar ${ }^{1}$ iD , José de Jesús Bohórquez- \\ Rivero $^{1}$ iD, Roberto Carlos Guzmán-Sáenz ${ }^{2}$ iD \\ ${ }^{1}$ Grupo de Investigación GIBACUS. Escuela de Medicina. Universidad del Sinú, Cartagena, Colombia. \\ ${ }^{2}$ Departamento de Medicina Interna. Universidad del Norte. Barranquilla, Colombia.
}

\section{RESUMEN}

Introducción: el SARS-CoV-2 representa al patógeno causante de la enfermedad denominada COVID-19. Dicha patología se ha extendido rápidamente a nivel mundial, constituyendo un importante problema de salud pública. Sus características clínicas no se limitan al tracto respiratorio, sino que también comprometen al sistema nervioso.

Objetivo: identificar los posibles mecanismos directos e indirectos por los cuales el SARS-CoV-2 afecta al sistema nervioso y describir los eventos fisiopatológicos responsables de las manifestaciones y complicaciones neurológicas reportadas en COVID-19.

Métodos: se realizó una revisión narrativa de la literatura encontrada en las bases de datos PubMed y Science Direct y en el motor de búsqueda Google Scholar utilizando las palabras claves. Se incluyeron artículos en idioma español e inglés publicados en el año 2020.

Resultados: se identificaron los posibles mecanismos de invasión directa del SARSCoV-2 al SNC (por las propiedades neurotrópicas del virus) y los mecanismos indirectos (derivados de las alteraciones metabólicas, el estado proinflamatorio y la desregulación del sistema inmune) detrás del compromiso neurológico asociado a COVID-19.

Conclusiones: la identificación de los posibles mecanismos que explican como el SARS-CoV-2 ingresa, se establece en el SNC y lo afecta directamente, así como su afectación indirecta, son un pilar fundamental para la comprensión de los eventos fisiopatológicos que explican las manifestaciones clínicas y complicaciones neurológicas reportadas en COVID-19.

Palabras Clave: COVID-19; manifestaciones neurológicas; infecciones por Coronavirus; neuropatología; citoquinas.

\section{ABSTRACT}

Introduction: SARS-CoV-2 represents the pathogen that causes the disease called COVID-19. This pathology has spread rapidly worldwide, constituting a major 
public health problem. Its clinical features are not limited to the respiratory tract, but also involve the nervous system.

Objective: to identify the possible direct and indirect mechanisms by which SARSCoV-2 affects the nervous system and to describe the pathophysiological events responsible for the neurological manifestations and complications reported in COVID-19.

Methodology: a narrative review of the literature found in the PubMed and Science Direct databases and in the Google Scholar search engine was carried out using the keywords. Articles in Spanish and English language published in 2020 were included.

Results: were identified the possible mechanisms of direct invasion of SARS-CoV2 to the CNS (due to the neurotropic properties of the virus) and the indirect mechanisms (derived from metabolic alterations, the pro-inflammatory state and the dysregulation of the immune system) behind the neurological compromise associated with COVID-19.

Conclusions: the identification of the possible mechanisms that explain how SARSCoV-2 enters, establishes itself in the CNS and affects it directly, as well as its indirect affectation, are a fundamental pillar for the understanding of the pathophysiological events that explain the clinical manifestations and neurological complications reported in COVID-19.

Keywords: COVID-19; neurological manifestations; Coronavirus infections; neuropathology; cytokines.

\section{INTRODUCCIÓN}

La enfermedad coronavírica de 2019 (COVID-19) causada por el Coronavirus-2 del Síndrome Respiratorio Agudo Severo (SARS-CoV-2), es una patología infectocontagiosa predominantemente respiratoria; no obstante, la evidencia creciente indica que la enfermedad no se limita al tracto respiratorio, sino que también puede afectar a otros sistemas, incluyendo el sistema nervioso. A medida que la enfermedad se propaga, las manifestaciones y complicaciones neurológicas en pacientes con COVID-19 se reportan cada vez con mayor frecuencia en la literatura científica (1). Dichos reportes plantean el posible potencial neurotrópico del SARS-CoV-2 (2). Tanto el sistema nervioso central (SNC) como el sistema nervioso periférico (SNP) son particularmente vulnerables ante las enfermedades inmunomediadas asociadas con infecciones y COVID-19 no ha sido la excepción (3).
La enzima convertidora de angiotensina 2 (ACE2) es un receptor de superficie de las células humanas y se ha demostrado que es utilizado por el SARS-CoV-2 para su ingreso a las células. La ACE2 actúa como el receptor de anclaje de la proteína en espiga (proteína S) del SARS-CoV-2 $(4,5,6)$. Al unirse la proteína $S$ a la ACE2 se desencadenan una serie de cambios conformacionales en su estructura, que permiten que sea escindida por la serina proteasa transmembrana de tipo 2 (TMPRSS2). La participación de la proteasa TMPRSS2 es fundamental debido a que la escisión de la proteína $\mathrm{S}$ asegura la fusión de la membrana viral con la membrana de la célula huésped. Tanto la ACE2 como la TMPRSS2 muestran una distribución generalizada en múltiples órganos (pulmones, corazón, cerebro, riñones, tracto gastrointestinal, entre otros) (7). Una vez el virus ingresa a la célula huésped, se activa la respuesta inmune y una respuesta inflamatoria inducida por citoquinas y quimiocinas. 
Se han evidenciado niveles significativamente elevados de estos mediadores proinflamatorios en pacientes con COVID-19 grave, este fenómeno se denomina "tormenta de citoquinas", es característico de esta etapa de la enfermedad y se ha asociado con la aparición del síndrome de dificultad respiratoria aguda (SDRA), sepsis y disfunción multiorgánica (8).

A la luz de la evidencia, han surgido nuevos hallazgos relacionados con COVID-19 y uno de ellos es el compromiso neurológico asociado a la enfermedad. Se han propuesto diversas teorías sobre la génesis de las manifestaciones y complicaciones neurológicas reportadas en los pacientes con infección por SARS-CoV-2/COVID-19. Se han postulado tanto mecanismos directos como indirectos. Los mecanismos directos hacen alusión al ingreso directo del virus al SNC que puede ser a través del receptor ACE2, diseminación hematógena, transporte neuronal retrógrado o anterógrado, o a través de la placa cribiforme y el bulbo olfatorio $(9,10,11)$. Por otro lado, los mecanismos indirectos hacen alusión a la respuesta inflamatoria, la respuesta inmune y la tormenta de citoquinas $(12,13,14)$.

La comprensión de los posibles mecanismos de neuroinvasión del SARS-CoV-2 y de sus implicaciones fisiopatológicas contribuye a los clínicos a identificar mejor las manifestaciones y complicaciones neurológicas de la infección y, de esta manera, se podría mejorar el abordaje y el tratamiento de la enfermedad. El objetivo de esta revisión es identificar los posibles mecanismos, directos e indirectos, por los cuales el SARS-CoV-2 afecta al sistema nervioso y describir los eventos fisiopatológicos responsables de las manifestaciones y complicaciones neurológicas reportadas en estos pacientes.

\section{MÉTODOS}

Se realizó una búsqueda bibliográfica en las bases de datos PubMed y Science Direct y en el motor de búsqueda Google Scholar utilizando los siguientes términos:

COVID-19, neurológicas, infecciones por Coronavirus, neuropatología, citoquinas. Se incluyeron artículos en idioma español e inglés publicados en el año 2020. La recolección de la información se realizó del 10 de Julio al 25 de septiembre de 2020. Se identificaron un total de 155 artículos entre artículos originales, revisiones de tema, revisiones sistemáticas, cartas al editor, reportes de caso y series de casos. Fueron seleccionados 62 artículos (5 en idioma español y 57 en idioma inglés) que se ajustaron al objetivo del artículo.

\section{RESULTADOS}

Mecanismos de ingreso del SARS-COV-2 al sistema nervioso

A lo largo de la historia, se han notificado eventos neurológicos secundarios a infecciones por otros coronavirus humanos (HcoV), como SARS-CoV, MERS-CoV, HCoV-229E, HCoV-OC43 y HCoVNL63 (15). El potencial neurotrópico de los $\mathrm{HCoV}$ se ha logrado demostrar a través de estudios in vitro e in vivo. Hoy por hoy, los mecanismos por los cuales el SARS-CoV-2 penetra en el sistema nervioso aún son intrincados y sus manifestaciones clínicas no se han establecido completamente. Sin embargo, al contemplar la fisiopatología conocida del SARS-CoV-2 y la similitud que exhibe dicho virus con los otros $\mathrm{HCoV}$, se han podido establecer hipótesis que ofrecen las explicaciones más probables $(3,9,16)$ (Figura 1).

Como se mencionó previamente, el SARS-CoV-2 utiliza la ACE2 como su receptor de entrada, y a la proteasa TMPRSS2 para el cebado de la proteína $S$ $(17,18)$. Los estudios cruzados de tejido humano de células positivas para ACE2 y TMPRSS2 encontraron la co-expresión de estas proteínas en las células epiteliales nasales y ciliadas, así como en células gliales, y neuronas (7). Por ello, se presume que la coexpresión de ACE2/TMPRSS2 en dichas células podría ser un medio de infiltración y/o proliferación del virus en el SNC (16).

Del mismo modo, los HCoV son capaces de ingresar al sistema nervioso por vía hematógena o transneuronal $(2,7,16)$. La diseminación hematógena 
hace alusión al paso del SARS-CoV-2 desde la circulación sistémica hasta la circulación cerebral, donde el enlentecimiento del flujo permite que el virus infecte las células endoteliales de la barrera hematoencefálica y obtenga acceso al SNC. La diseminación transneuronal se refiere a que el virus infecta una neurona periférica y se traslada al cuerpo celular neuronal en áreas cercanas del cerebro (9); este mecanismo es posible gracias a la polarización de las neuronas, propiedad que les da la capacidad de recibir y transferir información. Dicho transporte puede ser retrógrado o anterógrado y se ve facilitado por las proteínas dineína y kinesina, que también pueden ser objetivos del virus (19). Al ingresar el virus al SNC, existe evidencia de que se disemina a lo largo de las vías de los neurotransmisores, como el sistema del rafe dorsal serotoninérgico, o por vía hematógena a través de los espacios de VirchowRobin, y una vez establecido por completo es capaz de generar alteraciones neuronales (9).

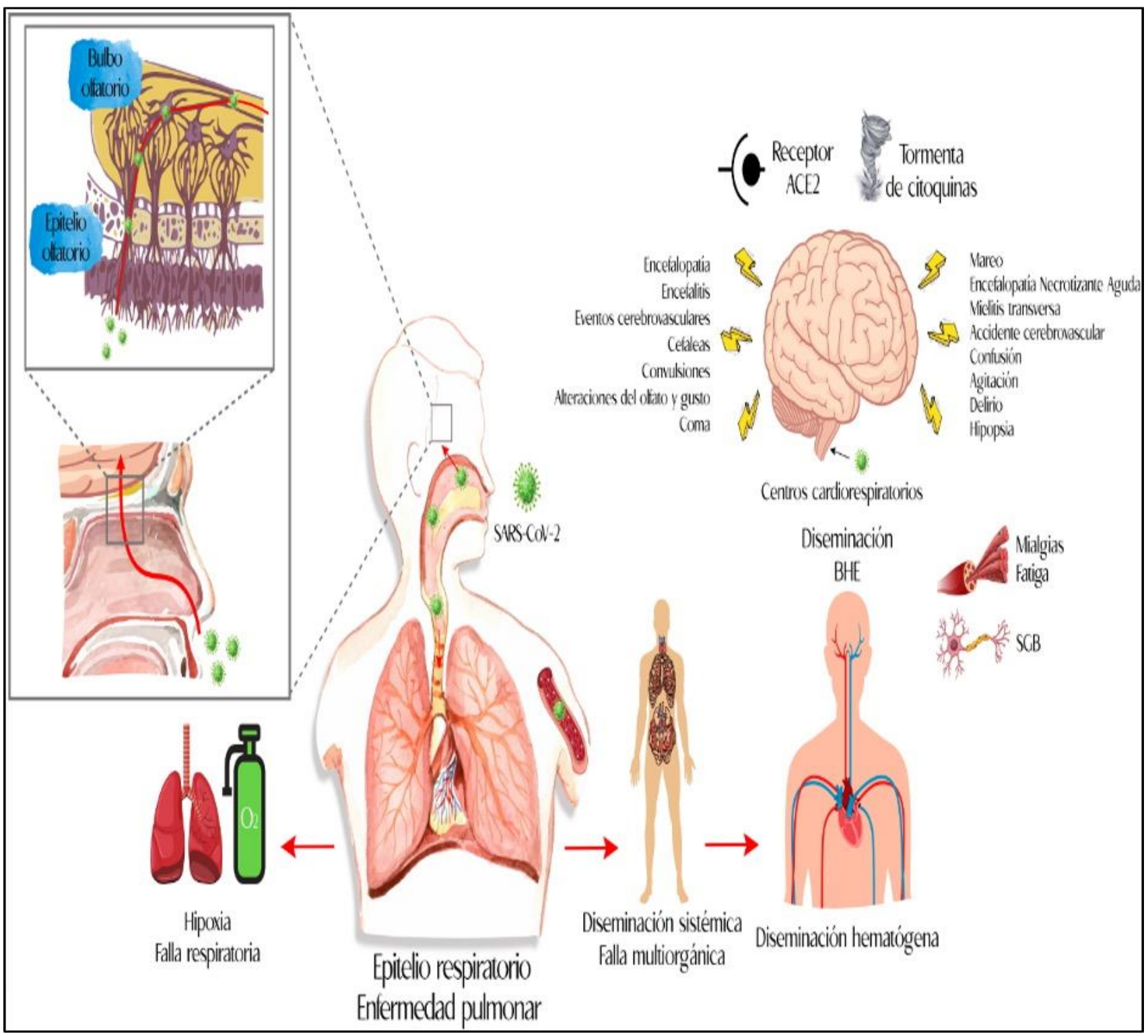

Figura 1. Principales mecanismos propuestos de neuroinvasión del SARS-CoV-2 y manifestaciones neurológicas de COVID19. ACE2: Enzima Convertidora de Angiotensina II; BHE: Barrera Hematoencefálica; SGB: Síndrome de Guillain-Barré. 
La invasión del SARS-CoV-2 al sistema nervioso a través de la placa cribiforme y el bulbo olfatorio puede actuar como una vía adicional (9). La vía olfatoria comienza en las neuronas receptoras olfatorias; estas son pequeñas neuronas bipolares cuyos cilios sensitivos se distribuyen ampliamente en el epitelio olfatorio de la cavidad nasal (están expuestos al SARS-CoV-2), desde allí sus axones se extienden y atraviesan la placa cribiforme, para finalizar en el bulbo olfatorio, donde hacen sinapsis con las células presentes en esta estructura. Posteriormente, el nervio olfatorio se divide en dos ramas y se dirige hacia el núcleo olfatorio presente en la corteza piriforme; el virus puede seguir esta ruta y posteriormente invadir áreas como el tronco encefálico y el tálamo (19).

El SARS-CoV-2 tiene una afinidad muy particular por las neuronas del tronco encefálico donde se encuentran los centros que controlan la dinámica respiratoria $(19,20,21)$. Los antígenos virales de otros $\mathrm{HCoV}$ ya han sido detectados en el tallo encefálico, especialmente en regiones que incluyen el núcleo ambiguo y el núcleo del fascículo solitario.

El núcleo del fascículo solitario recibe información sensorial de mecanorreceptores y quimiorreceptores pulmonares y del tracto respiratorio (detectan los cambios en las concentraciones de $\mathrm{CO}_{2}$ y $\mathrm{O}_{2}$ ); mientras que las fibras eferentes del núcleo ambiguo y del núcleo del fascículo solitario inervan a glándulas, músculo liso de la vía aérea y vasos sanguíneos.

Estas interconexiones neuroanatómicas podrían sugerir que la muerte de los pacientes puede deberse a disfunción de los centros cardiorrespiratorios en el tronco encefálico posterior a la entrada del SARSCoV-2 en dicha estructura, presumiendo que la falla respiratoria en COVID-19 podría tener un componente neurogénico $(19,20)$. El mecanismo descrito se relaciona con lo notificado por Li et al. (22), quienes reportaron una posible falla ventilatoria aguda de origen central en una paciente femenina de 24 años con diagnóstico de COVID-19, y la asocian con el potencial neuroinvasivo del SARS-CoV-2 (22).

\section{Mecanismo neuropatológico del daño del SNC}

\section{Lesión cerebral hipóxica}

El SARS-CoV-2 también podría ocasionar daño neurológico a través de mecanismos indirectos. El virus se replica y prolifera en los neumocitos y provoca un exudado inflamatorio alveolar e intersticial difuso, edema y formación de membranas transparentes; por lo tanto, el intercambio gaseoso a nivel alveolar se afecta significativamente $(15,21)$. Este hecho conduce a hipoxia en el SNC, y además se produce un incremento del metabolismo anaerobio con sus productos de desecho como el ácido láctico en las células del SNC, vasodilatación cerebral, edema celular e intersticial, obstrucción del suministro de sangre al cerebro, y cefalea debido a la isquemia y congestión posterior a la infección viral. Si la hipoxia continúa, la función cerebral empeora e incluso puede provocar coma o la muerte (23). La hipoxia severa también puede resultar en un evento cerebrovascular agudo, como un accidente cerebrovascular (ACV) isquémico agudo. También se ha demostrado que los pacientes con COVID-19 a menudo sufren de hipoxia severa y viremia, las cuales tienen el potencial de producir encefalopatía tóxica (24).

\section{Lesión inmunomediada}

La respuesta exagerada del sistema inmune ante la infección por SARS-CoV-2, ocasiona la liberación de una gran cantidad de citoquinas proinflamatorias como las interleucinas (IL) IL-2, IL-6, IL-7 e IL-10, el factor de necrosis tumoral $\alpha(\mathrm{TNF}-\alpha)$ y el factor estimulante de colonias de granulocitos (G-CSF) (25). La liberación de estos factores altera la permeabilidad de la barrera hematoencefálica (BHE) e incrementa la activación de rutas neuroinflamatorias. Además, algunas de estas citoquinas pueden potenciar la hiperexcitabilidad neuronal por medio de la activación de los receptores de glutamato y provocar convulsiones de forma aguda (26). Del mismo modo, se sugiere que la respuesta inmune e inflamatoria exagerada en la infección por SARS-CoV-2 puede provocar lesión inflamatoria y edema cerebral; este proceso conduce a alteraciones en el estado de conciencia de estos pacientes (27). Además, la evidencia sugiere que los $\mathrm{HCoV}$ pueden infectar a los leucocitos (linfocitos, 
granulocitos, monocitos y derivados de monocitos) e inducir su migración (28). Es decir, los leucocitos una vez activados por la infección, se diseminan hacia otros tejidos y cruzan la BHE para acceder al SNC; proceso que se denomina "Mecanismo de caballo de Troya" (29). En el SNC, los leucocitos producen citoquinas proinflamatorias como el TNF que pueden dañar oligodendrocitos y/o neuronas, y quimiocinas como CCL5, CXCL10 y CXCL11 que inducen la quimioatracción de células $\mathrm{T}$ activadas y/u otros leucocitos (29). Después de la infección, los astrocitos también pueden producir quimiocinas, incluidas CCL2, CCL5 y CXCL12, que participan en el reclutamiento de más leucocitos infectados. Por lo tanto, el SARS-CoV-2 podría iniciar un círculo vicioso neuroinflamatorio aberrante, que da lugar a neuropatología (1). La tormenta de citoquinas a nivel del SNC puede provocar la degradación y ruptura de la $\operatorname{BHE}(15,21)$.

La pérdida del olfato también puede obedecer a la respuesta inflamatoria en la cavidad nasal, que impide temporalmente que los olores estimulen a las neuronas receptoras olfativas. El daño a las neuronas olfativas requerirá un periodo más largo de regeneración de axones para hacer sinapsis exitosas con el bulbo olfatorio (30). Con respecto a la disfunción del sentido del gusto, la tormenta de citoquinas en pacientes con COVID-19 puede apuntar a las papilas gustativas y causar ageusia (31). Cabe resaltar que, en todos los sucesos previamente mencionados, el virus no tiene invasión directa ni desmielinización parainfecciosa (32).

Evidencias y reportes de las manifestaciones y complicaciones neurológicas asociadas a la infección por SARS-CoV-2/COVID-19

Existen dos series de casos que describen específicamente las manifestaciones neurológicas y las complicaciones en pacientes con COVID-19. La primera es una serie de casos retrospectiva y observacional realizada por Mao et al. (33) que incluyó 214 pacientes de Wuhan, China, con diagnóstico confirmado de COVID-19; los autores reportaron que el $36,4 \%$ de los pacientes presentaron alguna manifestación neurológica clasificada como afectación central $(24,8 \%)$, periférica $(10,7 \%)$ y musculoesquelética (10,7\%). Los principales síntomas reportados fueron mareos $(16,8 \%)$, cefalea $(13,1 \%)$, hipogeusia $(5,6 \%)$ e hiposmia $(5,1 \%)$; el $45.5 \%$ de estos pacientes correspondía a casos de infección grave. Los datos sugieren que los pacientes con enfermedad grave tenían más probabilidades de tener complicaciones neurológicas, como eventos cerebrovasculares agudos $(5,7 \%$ vs $0,8 \%)$, alteración del estado de conciencia $(14,8 \%$ vs $2,4 \%)$ y lesión del músculo esquelético (19,3\% vs $4.8 \%)$, en comparación con aquellos con formas leves de la infección (33). La segunda es una serie observacional de 58 pacientes en dos Unidades de Cuidados Intensivos en Estrasburgo, Francia; la mediana de edad de los pacientes fue de 63 años y las complicaciones neurológicas se observaron en un porcentaje significativo 49/58 (84\%); en esta serie, según lo evaluado por el método para la evaluación de la confusión en la unidad de cuidados intensivos (CAM-UCI), la agitación fue el síntoma más común 40/58 (69\%) seguido de confusión 26/40 (65\%). Los signos del tracto corticoespinal estuvieron presentes en 39/58 (67\%) y se observó un síndrome disejecutivo en el momento del alta en 14/39 (36\%) de los casos (34).

Las manifestaciones neurológicas y las complicaciones de la infección por SARS-CoV2/COVID-19 se pueden dividir en centrales y periféricas como se describe en la Tabla 1.

\section{Manifestaciones y complicaciones del SNC Cefalea y mareos}

Los dolores de cabeza y los mareos son síntomas inespecíficos de muchas enfermedades. Se han informado como síntomas leves asociados con la presentación de COVID-19 en diferentes reportes; su incidencia varía del 3 al 12,1\% (8,22,36). Sin embargo, el mecanismo específico y la fisiopatología no se discuten en ninguno de estos informes.

\section{Encefalopatía}

Mao et al. (33) reportaron cefalea y encefalopatía en el $40 \%$ de los pacientes de su cohorte; sin embargo, no se describieron los detalles ni los criterios diagnósticos utilizados. Del mismo modo, Filatov et 
al. (37) reportaron un caso de un hombre de 74 años con enfermedad de Parkinson y múltiples comorbilidades cardiovasculares y pulmonares, que consultó al servicio de urgencias por dificultad respiratoria, fiebre persistente, cefalea y alteración del estado de conciencia. La tomografía computarizada (TC) de cráneo carecía de alteraciones agudas; en el electroencefalograma se evidenciaron hallazgos de disfunción focal del lóbulo temporal izquierdo $\mathrm{y}$ focos de epileptogenicidad; el estudio del líquido cefalorraquídeo (LCR) se encontraba dentro de parámetros normales y no se logró aislar el virus. En base a estos hallazgos, se consideró que además de los síntomas respiratorios, el paciente tenía encefalopatía (37). Asimismo, Chen et al. (38) en un estudio retrospectivo de las características clínicas de 113 pacientes con COVID-19 realizado en China, documentaron encefalopatía hipóxica en 20 pacientes, con una incidencia significativamente menor en los pacientes que se habían recuperado (38).

Tabla 1. Manifestaciones y complicaciones neurológicas de la infección por SARS-CoV-2/COVID-19 (3,35).

\section{Localización}

Manifestaciones y complicaciones

\section{neurológicas}

\begin{tabular}{|c|c|}
\hline \multirow[t]{12}{*}{ Sistema Nervioso Central } & Cefalea \\
\hline & Mareo \\
\hline & Encefalopatía \\
\hline & Encefalopatía Necrotizante Aguda \\
\hline & Encefalitis \\
\hline & Convulsiones \\
\hline & Mielitis Transversa \\
\hline & Accidente cerebrovascular \\
\hline & Confusión \\
\hline & Agitación \\
\hline & Delirio \\
\hline & Coma \\
\hline \multirow[t]{6}{*}{ Sistema Nervioso Periférico } & Hipogeusia \\
\hline & Hiposmia \\
\hline & Síndrome de Guillain Barré \\
\hline & Neuralgia \\
\hline & Debilidad generalizada \\
\hline & Mialgias \\
\hline
\end{tabular}

\section{Encefalopatía necrotizante hemorrágica aguda}

Este tipo de encefalopatía es una complicación poco común de la influenza y otras infecciones virales, relacionada con la tormenta de citoquinas intracraneales (39), la cual también se ha evidenciado en pacientes con COVID-19 grave (28). Poyiadji et al. (32) reportaron un caso de un paciente con encefalopatía hemorrágica necrotizante aguda 
asociada a COVID-19, y diagnosticada a través de neuroimágenes (32). Se trataba de un paciente de 50 años que se presentó con historia de tres días de tos, fiebre y alteración del estado de conciencia. La prueba de reacción en cadena de la polimerasa (PCR) fue positiva para COVID-19 y negativa para el virus del herpes simple 1 y 2 , el virus del Nilo occidental y el virus de la varicela zóster. La TC de cráneo sin contraste demostró hipoatenuación simétrica dentro del tálamo medial bilateral, con una angiografía por TC y una venografía por TC normales. La resonancia magnética (RM) cerebral demostró lesiones que realzaban el borde hemorrágico dentro de los tálamos bilaterales, los lóbulos temporales mediales y las regiones subinsulares. El mecanismo propuesto fue que probablemente se deba a que la tormenta de citoquinas producida en el SNC da como resultado la interrupción de la barrera hematoencefálica y daño al parénquima cerebral (32).

\section{Encefalitis}

La encefalitis se define como la inflamación del parénquima cerebral, se evidencia clínicamente por la aparición de fiebre, vómitos, cefalea, alteración del estado de conciencia y convulsiones; se confirma por aislamiento viral en el LCR con evidencia de pleocitosis y por neuroimágenes que sugieran inflamación del parénquima cerebral ${ }^{40}$. Moriguchi et al. (11) reportaron el primer caso de meningoencefalitis asociada a COVID-19; se trataba de un joven japonés de 24 años quien presentó fiebre, episodios eméticos, alteración del estado de conciencia y convulsiones generalizadas. Posteriormente, se logró evidenciar el virus en el LCR y las neuroimágenes mostraron compromiso del lóbulo temporal mesial, ventrículo lateral e hipocampo (11). En otro reporte de caso de Wuhan, China; Ye et al. (27) notificaron que se puede encontrar encefalitis autolimitada, junto con mialgias, signos de irritación meníngea, fiebre y alteración del estado de conciencia en pacientes con COVID-19; aunque el diagnóstico definitivo de encefalitis viral depende en gran medida del aislamiento del virus, esto es difícil para COVID-19 porque la diseminación del SARS-CoV-2 es transitoria y su título en LCR es muy bajo. De manera constante, los anticuerpos anti-SARS-CoV-
2 IgM e IgG no fueron detectables en la muestra de LCR del paciente (27); sin embargo, de manera interesante, los modelos animales han demostrado que las vías neuroinvasivas pueden causar una pérdida neuronal en ausencia de encefalitis (41).

\section{Mielitis transversa aguda}

La mielitis transversa se caracteriza por una inflamación aguda o subaguda de la médula espinal que ocasiona déficits neurológicos potencialmente incapacitantes, como debilidad motora y sensorial, así como disfunción autonómica. La mielitis transversa aguda se ha asociado con una amplia gama de etiologías, que se han subdividido en mielopatías compresivas y no compresivas. Las etiologías no compresivas incluyen infecciosas, autoinmunes, isquémicas, paraneoplásicas, efectos de radiación, post-vacunación y post-infecciosas, así como causas idiopáticas. El diagnóstico se basa en los hallazgos clínicos característicos, además de los estudios serológicos, de resonancia magnética y de LCR (42,43). Zhao et al. (44) reportaron un caso de mielitis aguda en un hombre de 66 años de Wuhan, China que presentaba fiebre y dolores corporales. Durante el ingreso desarrolló parálisis flácida aguda bilateral de miembros inferiores, nivel sensitivo en $\mathrm{T}-10$ con incontinencia urinaria e intestinal. La TC de tórax confirmó una neumonía atípica y la PCR de secreción nasofaríngea fue positiva para COVID-19. La serología para los demás microorganismos fue negativa. Los autores atribuyeron la mielitis aguda a la tormenta de citoquinas y la respuesta inflamatoria hiperactiva, como lo evidencian los altos niveles de ferritina sérica, proteína $\mathrm{C}$ reactiva, amiloide A sérico e IL-6 (44). Se han notificado reportes de casos similares que vinculan al COVID-19 con la mielitis aguda como una complicación neurológica $(45,46)$. Además, se ha publicado un caso de mielitis necrotizante aguda posterior a COVID-19 (42).

\section{Enfermedad cerebrovascular aguda}

La enfermedad cerebrovascular comprende el ACV isquémico y hemorrágico. Mao et al. (33) comunicaron 6 casos de ACV en su cohorte de 214 pacientes; reportaron 5 casos de ACV isquémico y 1 caso de ACV hemorrágico (33). La cohorte francesa tuvo 3 casos de ACV isquémico que se detectaron a 
través de neuroimagenes cuando los pacientes se sometieron a estas por exhibir características encefalopáticas (34). Los pacientes no presentaban signos neurológicos focales. Probablemente los síntomas estaban enmascarados por la presencia de encefalopatía, pero se resalta la importancia de las neuroimágenes en la evaluación de estos casos; sin embargo, los autores señalaron que se necesitan más pruebas para establecer una relación causal entre ACV y COVID-19 (34).

Sharifi et al. (47) reportaron un caso de ACV hemorrágico en un varón de 79 años de edad con COVID-19 positivo. Ingresó al servicio de urgencias con una escala de coma de Glasgow 7/15 y antecedentes de fiebre y tos. En el examen físico se encontraron reflejos plantares extensores bilaterales positivos, al examen pulmonar se evidenciaron crépitos gruesos en la base pulmonar izquierda. La PCR de secreción nasofaríngea fue positiva para COVID-19. La TC de tórax mostró opacidad en patrón de vidrio esmerilado sugestiva de neumonía viral. La TC de cráneo reveló una hemorragia masiva en el hemisferio derecho con extensión intraventricular y subaracnoidea. El paciente no era hipertenso conocido ni tomaba ningún anticoagulante que pudiera haber causado este evento. Las plaquetas y el TP/INR al ingreso fueron normales. Los autores postularon que probablemente la desregulación en los receptores ACE2 conduzca a la autorregulación cerebral, el sistema simpáticoadrenal y el flujo sanguíneo cerebral podrían haber provocado la hemorragia (47). En este sentido, la unión del SARS-CoV-2 a los receptores ACE2 también adquiere importancia en los casos de hemorragia intracerebral, la cual puede explicarse debido a la presencia de receptores ACE2 en el endotelio vascular cerebral y su función autorreguladora, que se reduce cuando es invadido por el virus, causando elevación de la presión arterial cerebral y produciendo como consecuencia la ruptura de los vasos sanguíneos $(19,48)$. En los casos de accidente cerebrovascular isquémico, los posibles mecanismos incluyen la hipercoagulabilidad asociada con la inflamación, la activación plaquetaria, la deshidratación y la cardioembolia por lesión cardíaca relacionada con el virus; que causan hipoxemia y puede empeorar aún más el daño neuronal (49).

\section{Otras manifestaciones del SNC}

Mao et al. (33) también informaron neuralgia en 5 pacientes y epilepsia y ataxia en cada uno de ellos, pero no se mencionaron más detalles (33). Como se ha mencionado, COVID-19 se ha asociado a actividad epileptógena $(0.5 \%)$, lo que podría indicar injuria directa en lóbulo temporal a causa de encefalitis (9). Sin embargo, se requieren más investigaciones para esclarecer los mecanismos específicos detrás de dicha manifestación. Asimismo, la ataxia ya ha sido previamente descrita en infecciones por $\mathrm{HCoV}$ (48).

La alteración del estado de conciencia es un término general con varios mecanismos subyacentes. En pacientes con COVID-19, los posibles mecanismos incluyen infecciones, daño parenquimatoso, desequilibrio hidroelectrolítico, encefalopatías hipóxicas, tóxicas y metabólicas y estado epiléptico no convulsivo (49).

Es importante mencionar que, además de ser aislado en lágrimas, existe evidencia de que el SARS-CoV2 puede comprometer la conjuntiva, lo que a su vez podría ser el origen de la hipopsia (disminución de la agudeza visual), síntoma que también refieren algunos pacientes. Aunque, actualmente, la lesión retiniana y del nervio óptico solo se ha demostrado asociada a infección por otros HcoV (50).

\section{Manifestaciones y complicaciones del SNP}

\section{Disfunción quimiosensorial}

Yan et al. (51) documentaron disfunción quimiosensorial en 59 pacientes COVID-19 positivos y 203 COVID-19 negativos de un solo centro de EE.UU mediante una encuesta transversal realizada mediante Internet. Se evidenció que la disfunción del olfato y el gusto era mayor en los casos positivos de COVID-19 en comparación con los casos negativos (pérdida del olfato: $68 \%$ vs. $16 \%$ y pérdida del gusto: $71 \%$ vs. $17 \%$ ). La mayoría de los pacientes de este estudio eran ambulatorios, no necesitaron hospitalización y ninguno requirió ventilación mecánica. Por lo tanto, teorizaron que 
probablemente en los pacientes ambulatorios con COVID-19 el virus se propaga por vía nasal en comparación con los pacientes críticamente enfermos en los que la propagación es probablemente pulmonar (51). Del mismo modo, Bagheri et al. (52) notificaron los resultados de una gran cohorte Iraní que incluyó 10.069 pacientes mediante el empleo de una encuesta basada en un cuestionario en línea. Los participantes fueron casos con disminución reciente del sentido del olfato (dentro de las últimas 4 semanas del inicio del brote de COVID 19 en Irán). El 48,23\% de los encuestados solo reportaron anosmia e hiposmia, mientras que el $83,38 \%$ también tenían hipogeusia. El inicio de la anosmia fue repentino en el 76,24\%. Otras características clínicas informadas por los participantes fueron síntomas de gripe o resfriado antes de la anosmia (75,5\%), dolores de cabeza $(48,6 \%)$, congestión nasal $(43,7 \%)$ y fiebre $(37,3 \%)$ (52). En contraste, en la cohorte presentada por Mao et al. (33) informaron alteración del olfato en 11 pacientes $(5,1 \%)$ y del gusto en 12 (5,6\%) (33). Mientras que, en la cohorte francesa de pacientes con COVID-19 no se informó pérdida del olfato y del gusto (34). Los posibles mecanismos de estas alteraciones han sido previamente descritos en el presente artículo.

\section{Síndrome de Guillain-Barré}

El síndrome de Guillain-Barré (SGB) es la causa más frecuente de parálisis flácida en los países desarrollados. Dicho trastorno neurológico es una polineuropatía aguda mixta, desmielinizante y axonal que puede aparecer en cualquier edad; no obstante, es más frecuente en la infancia. Aunque su etiopatogenia no está totalmente aclarada, abarca fenómenos inmunológicos responsables de la destrucción de la mielina de los nervios periféricos. Los fenómenos inflamatorios anómalos pueden estar desencadenados por agentes infecciosos, tóxicos, bioquímicos o en el contexto de una enfermedad tumoral (53). Zhao et al. (44) reportaron el compromiso del SNP con el desarrollo del síndrome parainfeccioso de Guillain-Barré en una mujer de 61 años; sin embargo, los autores reconocen el hecho de que la infección por SARS-CoV-2 en su caso podría ser una coincidencia más que una causalidad (44).
Del mismo modo, Virani et al. (54) informaron de SGB en un varón de 54 años de EE.UU que presentó una parálisis ascendente de rápida progresión que le provocó dificultad respiratoria. No hubo disfunción vesical o intestinal. Los reflejos estaban ausentes y la RM de columna era normal. Tenía antecedentes de diarrea que precedieron al ataque agudo de debilidad y dio positivo para COVID-19 (54). Posteriormente, Sedaghat et al. (55) informaron sobre un hombre Iraní de 61 años con diabetes mellitus, que presentaba tos, fiebre y, a veces, disnea, dos semanas antes de presentar parálisis ascendente que conducía a cuadriplejía y parálisis facial bilateral. El estudio de conducción nerviosa y la electromiografía (NCS/EMG) sugirieron neuropatía motora sensitiva axonal aguda. Los autores sugirieron que el SGB debe considerarse como una complicación neurológica del COVID-19 ya que la afectación respiratoria es común en el COVID-19 y puede ser un factor de riesgo para el desarrollo del SGB (55). El mecanismo sugerido es el mimetismo molecular en el que el patógeno probablemente comparte epítopos similares a los componentes de los nervios periféricos. Los anticuerpos producidos por el sistema inmunológico del huésped para combatir el virus reaccionan de forma cruzada y se unen a los nervios periféricos causando disfunción neuronal (49).

Seguidamente, Toscano et al. (56) describieron la presencia de SGB como complicación neurológica en 5 pacientes con infección por SARS-CoV-2 en Italia, con un intervalo de 5-10 días entre los síntomas respiratorios y los síntomas del SGB. Entre estos pacientes, se informaron déficits severos, afectación axonal e insuficiencia respiratoria con la consiguiente necesidad de ventilación mecánica (56).

\section{Lesión del músculo esquelético}

Mao et al. (33) comunicaron lesión del músculo esquelético en 17 pacientes (19,3\%) dentro del grupo de pacientes con enfermedad grave, y 6 pacientes $(4,8 \%)$ en el grupo no grave. Definieron la lesión del músculo esquelético como paciente con mialgia y un nivel elevado de creatina quinasa $(\mathrm{CK})$ sérica por encima de $200 \mathrm{U} / \mathrm{L}$. Concluyeron que no estaba claro 
si se debía al efecto directo del virus sobre el tejido muscular (33), pero como se ha mencionado previamente, similar al SARS-CoV-1, el SARSCoV-2 tiene la capacidad de penetrar en las células que expresan los receptores ACE2, dado que la ACE2 se expresa en las células musculares; por lo tanto, se debe considerar esta ruta de invasión como explicación al daño muscular que exhiben estos pacientes (49). Del mismo modo, en la cohorte de Mao et al. (33), pacientes con lesión muscular tenían niveles más altos de proteína $\mathrm{C}$ reactiva y de dímero $\mathrm{D}$, manifestaciones de hiperinflamación y coagulopatía asociada; por lo cual el otro posible mecanismo propuesto fue el daño muscular inmunomediado. Sin embargo, es importante señalar que los pacientes en el grupo de enfermos graves, además de las enzimas musculares elevadas, también tenían enzimas hepáticas elevadas y función renal alterada lo que podría haber contribuido a este cuadro clínico. Además, no se realizó ningún estudio diagnóstico específico para confirmación como NCS/EMG o histopatología muscular; por lo tanto, es difícil excluir que estos pacientes puedan tener miopatía y neuropatía por enfermedades críticas además del daño del músculo esquelético (33).

La injuria muscular con el incremento en los niveles de CK, mioglobina y lactato deshidrogenasa se ha evidenciado en pacientes con forma grave de la enfermedad; y su presencia toma importancia ya que se conoce que otra posible complicación de la COVID-19 es la rabdomiólisis (57). Esto se sugirió basado en que Jin et al. (57) reportaron un caso de rabdomiólisis en un hombre de 60 años con diagnóstico de COVID-19. Los autores indicaron que se debe considerar el diagnóstico de rabdomiólisis cuando los pacientes manifiestan dolor muscular focal y fatiga (57).

\section{Otras manifestaciones del SNP}

Las implicaciones del SNP también han sido evidenciadas en dos pacientes que fueron diagnosticados con síndrome de Miller-Fisher y polineuritis craneal entre los tres a cinco días posteriores a la presencia de síntomas asociados con COVID-19 (58). La parálisis de Bell también ha sido reportada. Se trató de una mujer de 65 años que fue ingresada en un hospital Chino por una caída facial izquierda precedida por un historial de dos días de dolor en la región mastoidea. No tenía fiebre, tos ni síntomas respiratorios previos. El examen físico mostró parálisis del nervio facial de la motoneurona inferior izquierda. La RM cerebral no mostró anormalidades; sin embargo, la PCR de secreción nasofaríngea resultó positiva para COVID-19 y la TC de tórax reveló patrón de vidrio esmerilado en la base del pulmón derecho. La parálisis facial izquierda se resolvió espontáneamente con el pasar del tiempo (59).

\section{Abordaje diferencial}

El abordaje diferencial de las manifestaciones neurológicas en estos tiempos puede representar un verdadero desafío para el personal de atención médica. Es decir, se puede dificultar la distinción entre causalidad verdadera y concomitancia no etiológica, lo cual adquiere especial importancia al presentarse reportes de casos con manifestaciones neurológicas atribuibles a COVID-19 (3).

Partiendo de este hecho y a pesar de la poca evidencia existente, es imprescindible que el personal de atención médica conozca las observaciones clínicas con las que actualmente se cuenta, con el fin de que les permita trazar una guía en la atención de los pacientes con clínica neurológica. Por ejemplo, la cefalea podría ser un síntoma de compromiso meníngeo especialmente si se acompaña de rigidez de nuca; esta observación clínica resulta interesante dado que la mayoría de las meningitis virales no presentan rigidez de nuca y este signo ha sido observado en meningitis por SARS-CoV-2 (20). De igual manera, en otros estudios publicados se ha notificado la aparición de dolores de cabeza aislados, es decir, sin la presencia de otros síntomas de tipo neurológico. Este hecho podría sugerir que el mecanismo más probable obedezca a una enfermedad subyacente del paciente que a una invasión primaria del SARS-CoV-2 en el SNC. Otro ejemplo puede ser que, de manera característica, la hiposmia e hipogeusia en los pacientes con infección por SARS-CoV-2 no se acompañan de obstrucción nasal, ni otras manifestaciones de rinitis (3). Por ello, tanto los 
antecedentes del paciente como una buena anamnesis y una correcta evaluación semiológica son una herramienta fundamental al momento de abordar estos pacientes.

El ACV representa un tópico importante en medio de la actual pandemia. La asociación aparente entre COVID-19 grave y accidente cerebrovascular se debe probablemente al hecho de que ambas afecciones comparten factores de riesgo similares y actualmente no se ha esclarecido si la infección per $s e ́$ representa un factor de riesgo independiente de accidente cerebrovascular (9).

Cabe mencionar que, independientemente de cualquier manifestación neurológica, sea directa o indirecta, la pandemia de COVID-19 ha ejercido gran impacto en el abordaje de pacientes neurológicos, ya sea infectados o no. La mayoría de las instituciones a nivel mundial han sido influenciadas negativamente en términos de prestación de servicios, restricciones de movilidad y miedo a acceder a servicios de salud (3). En este sentido, en las clínicas neurológicas, la atención electiva de pacientes no está ampliamente disponible y se han priorizado los casos urgentes. Debido a esto, el uso de telemedicina es una necesidad creciente y actualmente se han desarrollado guías para el manejo de COVID-19 para dicho propósito (60).

En varias instituciones se ha implementado el llamado "código de accidente cerebrovascular protegido". Dicho código se origina bajo la premisa de que cada paciente con accidente cerebrovascular está potencialmente infectado y debe tratarse en consecuencia. Sin embargo, mantener los altos estándares de cuidado del accidente cerebrovascular es de suma importancia. Por ejemplo, se deben realizar las neuroimágenes rápidamente para evitar retrasos innecesarios $(61,62)$. Inicialmente, en la presentación del paciente con posible accidente cerebrovascular, los médicos deben preguntar a los pacientes y/o a sus acompañantes si tienen síntomas indicativos de COVID-19. Si la respuesta es afirmativa, podrían considerar pedir una tomografía computarizada del tórax, que puede realizarse de manera simultánea con la tomografía computarizada de cráneo. En el proceso de admisión, los médicos deben establecer un plan de alta y realizar en el hospital solo las pruebas diagnósticas más importantes, con el fin de acortar la duración de la hospitalización. Finalmente, se debe solicitar interconsulta con un especialista en medicina interna o infectología en casos de sospecha de infección por SARS-CoV-2 o un especialista en medicina intensiva, si el paciente requiere altas fracciones de oxígeno inspirado. Cabe mencionar que los pacientes con accidente cerebrovascular con infección confirmada por SARS-CoV-2 deben ser transferidos a salas dedicadas de COVID-19. El abordaje de estos pacientes requiere de equipo multidisciplinario y la cooperación entre especialistas es esencial (3).

\section{CONCLUSIONES}

La identificación de los posibles mecanismos que explican como el SARS-CoV-2 ingresa, se establece en el SNC y lo afecta directamente, así como su afectación indirecta, son un pilar fundamental para la comprensión de los eventos fisiopatológicos que explican las manifestaciones clínicas y complicaciones neurológicas en estos pacientes.

CONFLICTOS DE INTERESES: Los autores no declaran conflictos de interés.

\section{REFERENCIAS}

1. Pezzini A, Padovani A. Lifting the mask on neurological manifestations of COVID-19. Nat Rev Neurol. 2020; doi: $10.1038 / \mathrm{s} 41582-020-0398-3$

2. Vergara JP, Tolosa C. Covid 19: manifestaciones neurológicas. Acta Neurol Colomb. 2020; vol.36, suppl.1, pp.7-10. ISSN 0120-8748. doi: 10.22379/24224022288

3. Tsivgoulis G, Palaiodimou L, Katsanos AH, Caso V, Köhrmann M, Molina C, et al. Neurological manifestations and implications of COVID-19 pandemic. Ther Adv Neurol Disord. 2020; 13:1756286420932036. $10.1177 / 1756286420932036$

4. Wu F, Zhao S, Yu B, Chen YM, Wang W, Song ZG, et al. A new coronavirus associated with human respiratory 
disease in China. Nature. 2020; 579(7798):265-269. doi: $10.1038 / \mathrm{s} 41586-020-2008-3$

5. DosSantos MF, Devalle S, Aran V, Capra D, Roque NR, Coelho-Aguiar JM, et al. Neuromechanisms of SARSCoV-2: A Review. Front Neuroanat. 2020;14(37):1-12. DOI: $10.3389 /$ fnana.2020.00037

6. Gheblawi M, Wang K, Viveiros A, Nguyen Q, Zhong JC, Turner AJ, et al. Angiotensin-Converting Enzyme 2: SARS-CoV-2 Receptor and Regulator of the ReninAngiotensin System: Celebrating the 20th Anniversary of the Discovery of ACE2. Circ Res. 2020; doi: 10.1161/CIRCRESAHA

7. Baig AM, Khaleeq A, Ali U, Syeda H. Evidence of the COVID-19 Virus Targeting the CNS: Tissue Distribution, Host-Virus Interaction, and Proposed Neurotropic Mechanisms. ACS Chem Neurosci. 2020; 11(7):995-998. doi: 10.1021/acschemneuro.0c00122

8. Pennisi M, Lanza G, Luca Falzone, Fisicaro F, Ferri R, Bella R. SARS-CoV-2 and the Nervous System: From Clinical Features to Molecular Mechanisms. Int J Mol Sci. 2020; 21(15): 5475. DOI: 10.3390/ijms21155475

9. Montalvan V, Lee J, Bueso T, De Toledo J, Rivas K. Neurological manifestations of COVID-19 and other coronavirus infections: A systematic review. Clinical Neurology and Neurosurgery. 2020; 194, 105921. doi: 10.1016/j.clineuro.2020.105921

10. Brann DH, Tsukahara T, Weinreb C, Lipovsek M, Van den Berge K, Gong B, et al. Non-neuronal expression of SARS-CoV-2 entry genes in the olfactory system suggests mechanisms underlying COVID-19-associated anosmia. Sci Adv. 2020; 6(31): eabc5801. doi: 10.1126/sciadv.abc5801

11. Moriguchi T, Harii N, Goto J, Harada D, Sugawara H, Takamino J. A first case of meningitis/encephalitis associated with SARS-Coronavirus-2. Int J Infect Dis. 2020; 94:55-58. doi: 10.1016/j.ijid.2020.03.062

12. Harapan H, Itoh N, Yufika A, Winardi W, Keam S, Te $\mathrm{H}$, et al. Coronavirus disease 2019 (COVID-19): A literature review. J Infect Public Health. 2020; 13(5):667-673. doi: 10.1016/j.jiph.2020.03.019

13. Abuabara E, Bohórquez J, Restom J, Sáenz J, Correa J, Mendoza C. Consideraciones actuales de antimaláricos en la infección por SARS-CoV-2 y su impacto. Rev.
Colomb. Nefrol. 2020; doi: 10.22265/acnef.7.Supl\%202.406

14. Sachdev K, Agrawal S, Ish P, Gupta N, Raheja K. Neurological manifestations of COVID-19: A brief review. Indian J Med Res. 2020; (1\&2):41-47. doi: 10.4103/ijmr.IJMR_1395_20

15. Carod-Artal FJ. Complicaciones neurológicas por coronavirus y COVID-19. Rev Neurol 2020; 70 (09):311-322 doi: 10.33588/rn.7009.2020179

16. Aghagoli G, Gallo Marin B, Katchur NJ, Chaves-Sell F, Asaad WF, Murphy SA. Neurological Involvement in COVID-19 and Potential Mechanisms: A Review. Neurocrit Care. 2020; doi: 10.1007/s12028-020-010494

17. Abduljalil JM, Abduljalil BM. Epidemiology, genome, and clinical features of the pandemic SARS-CoV-2: a recent view. New Microbes New Infect. 2020; 35:100672. doi: 10.1016/j.nmni.2020.100672

18. Deng YY, Zheng Y, Cai GY, Chen XM, Hong Q. Single-cell RNA sequencing data suggest a role for angiotensin-converting enzyme 2 in kidney impairment in patients infected with 2019-novel coronavirus. Chin Med J. 2020; 133(9):1129-1131. doi: 10.1097/CM9.0000000000000783

19. Conde Cardona G, Quintana Pájaro LD, Quintero Marzola ID, Ramos Villegas Y, Moscote Salazar LR. Neurotropism of SARS-CoV 2: Mechanisms and manifestations. J Neurol Sci. 2020; 412:116824. doi: 10.1016/j.jns.2020.116824

20. Moreno Zambrano D, Arévalo Mora M , Freire Bonifacini A, García Santibáñez R, Santibáñez Vásquez R. Manifestaciones Neurológicas Asociadas a la Infección Por SARS-CoV-2: Una Neuro-Revisión de COVID-19. Rev Ecuat Neurol. 2020; 29 (1): 115-124.

21. Orozco-Hernández JP, Marin-Medina DS, SánchezDuque JA. Manifestaciones neurológicas de la infección por SARS-CoV-2 [Neurological manifestations of SARS-CoV-2 infection]. Semergen. 2020; S11383593(20)30143-X. doi: 10.1016/j.semerg.2020.05.004

22. Li YC, Bai WZ, Hashikawa T. The neuroinvasive potential of SARS-CoV2 may play a role in the respiratory failure of COVID-19 patients. J Med Virol. 2020. doi: $10.1002 / \mathrm{jmv} .25728$ 
23. Zanin L, Saraceno G, Panciani PP, Renisi G, Signorini $\mathrm{L}$, Migliorati K, et al. SARS-CoV-2 can induce brain and spine demyelinating lesions. Acta Neurochir. 2020; 162(7):1491-1494. doi:10.1007/s00701-020-04374-x

24. Guo YR, Cao QD, Hong ZS, Tan YY, Chen SD, Jin HJ. The origin, transmission and clinical therapies on coronavirus disease 2019 (COVID-19) outbreak - an update on the status. Mil Med Res. 2020; 13;7(1):11. doi: 10.1186/s40779-020-00240-0

25. Yang X, Yu Y, Xu J, Shu H, Xia J, Liu H. Clinical course and outcomes of critically ill patients with SARSCoV-2 pneumonia in Wuhan, China: a single-centered, retrospective, observational study. Lancet Respir Med. 2020; (5):475-481. doi: 10.1016/S2213-2600(20)300795

26. Karimi N, Sharifi Razavi A, Rouhani N. Frequent Convulsive Seizures in an Adult Patient with COVID19: A Case Report, Iran Red Crescent Med J. 2020; 22(3): e102828. doi: 10.5812/ircmj.102828

27. Ye M, Ren Y, Lv T. Encephalitis as a clinical manifestation of COVID-19. Brain Behav Immun. 2020; 945-946. doi: 10.1016/j.bbi.2020.04.017

28. Mehta P, McAuley DF, Brown M, Sanchez E, Tattersall RS, Manson JJ; HLH Across Speciality Collaboration, UK. COVID-19: consider cytokine storm syndromes and immunosuppression. Lancet. 2020; 1033-1034. doi: 10.1016/S0140-6736(20)30628-0

29. Huang J, Zheng M, Tang X, Chen Y, Tong A, Zhou L. Potential of SARS-CoV-2 to Cause CNS Infection: Biologic Fundamental and Clinical Experience. Front Neurol. 2020; 11: 659. doi: 10.3389/fneur.2020.00659

30. Soler ZM, Patel ZM, Turner JH, Holbrook EH. A primer on viral-associated olfactory loss in the era of COVID19. Int Forum Allergy Rhinol. 2020; 10(7):814-820. doi: 10.1002/alr.22578

31. Vaira LA, Salzano G, Fois AG, Piombino P, De Riu G. Potential pathogenesis of ageusia and anosmia in COVID-19 patients. Int Forum Allergy Rhinol. 2020; 10(9):1103-1104. doi: 10.1002/alr.22593

32. Poyiadji N, Shahin G, Noujaim D, Stone M, Patel S, Griffith B. COVID-19-associated Acute Hemorrhagic Necrotizing Encephalopathy: Imaging Features. Radiology. 2020; 296(2): E119-E120. doi: 10.1148/radiol.2020201187
33. Mao L, Jin H, Wang M, Hu Y, Chen S, He Q, et al. Neurologic Manifestations of Hospitalized Patients with Coronavirus Disease 2019 in Wuhan, China. JAMA Neurol. 2020; 77(6):1-9. doi: 10.1001/jamaneurol.2020.1127

34. Helms J, Kremer S, Merdji H, et al. Neurologic Features in Severe SARS-CoV-2 Infection. N Engl J Med. 2020;382(23):2268-2270. doi:10.1056/NEJMc2008597

35. Ahmad I, Rathore FA. Neurological manifestations and complications of COVID-19: A literature review. J Clin Neurosci. 2020; 77:8-12. DOI: 10.1016/j.jocn.2020.05.017

36. Deng Y, Liu W, Liu K, Fang YY, Shang J, Zhou L. Clinical characteristics of fatal and recovered cases of coronavirus disease 2019 in Wuhan, China: a retrospective study. Chin Med J. 2020; 133(11):12611267. doi: $10.1097 / \mathrm{CM} 9.0000000000000824$

37. Filatov A, Sharma P, Hindi F, et al. Neurological Complications of Coronavirus Disease (COVID-19): Encephalopathy. Cureus. 2020; 12(3): e7352. doi: $10.7759 /$ cureus. 7352

38. Chen T, Wu D, Chen H, Yan W, Yang D, Chen G. Clinical characteristics of 113 deceased patients with coronavirus disease 2019: retrospective study. BMJ. 2020; 368:m1091. doi: 10.1136/bmj.m1091

39. Jasti M, Nalleballe K, Dandu V, Onteddu S. A review of pathophysiology and neuropsychiatric manifestations of COVID-19. J Neurol. 2020; 1-6. doi: 10.1007/s00415-020-09950-w

40. Ellul MA, Benjamin L, Singh B, Lant S, Michael BD, Easton A, et al. Neurological associations of COVID-19. Lancet Neurology. 2020; 19(9):767-783. doi: 10.1016/S1474-4422(20)30221-0

41. Saleki K, Banazadeh M, Saghazadeh A, Rezaei N. The involvement of the central nervous system in patients with COVID-19. Rev Neurosci. 2020; 31(4):453-456. doi: 10.1515/revneuro-2020-0026

42. Sotoca J, Rodríguez-Álvarez J. COVID-19-associated acute necrotizing myelitis. Neurol Neuroimmunol Neuroinflamm. 2020; 7 (5) e803; doi: 10.1212/NXI.0000000000000803

43. Durrani M, Kucharski K, Smith Z, Fien S. Acute Transverse Myelitis Secondary to Severe Acute 
Respiratory Syndrome Coronavirus 2 (SARS-CoV-2): A Case Report. Clin Pract Cases Emerg Med. 2020; 4(3):344-348. doi: 10.5811/cpcem.2020.6.48462

44. Zhao H, Shen D, Zhou H, Liu J, Chen S. Guillain-Barre syndrome associated with SARS-CoV-2 infection: causality or coincidence?. Lancet Neurol. 2020; doi: 10.1016/S1474-4422(20)30109-5

45. AlKetbi R, AlNuaimi D, AlMulla M, et al. Acute myelitis as a neurological complication of Covid-19: A case report and MRI findings. Radiol Case Rep. 2020;15(9):1591-1595. Published 2020 Jun 6. doi: 10.1016/j.radcr.2020.06.001

46. Sarma D, Bilello LA. A Case Report of Acute Transverse Myelitis Following Novel Coronavirus Infection. Clin Pract Cases Emerg Med. 2020;4(3):321323. doi:10.5811/cpcem.2020.5.47937

47. Sharifi-Razavi A, Karimi N, Rouhani N. COVID-19 and intracerebral haemorrhage: causative or coincidental?. New Microbes New Infect. 2020; 35:100669. doi: 10.1016/j.nmni.2020.100669

48. Fan H, Tang X, Song Y, Liu P, Chen Y. Influence of COVID-19 on Cerebrovascular Disease and its Possible Mechanism. Neuropsychiatr Dis Treat. 2020; 16:13591367. doi: 10.2147/NDT.S251173

49. Sharifian-Dorche M, Huot P, Osherov M, Wen D, Saveriano A, Giacomini PS, et al. Neurological complications of coronavirus infection; a comparative review and lessons learned during the COVID-19 pandemic. J Neurol Sci. 2020. 15; 417: 117085. doi: 10.1016/j.jns.2020.117085

50. Trejos-Gabriel JM. Stroke as a complication and prognostic factor of COVID-19. Neurologia. 2020; 35(5):318-322. doi: 10.1016/j.nrl.2020.04.01529

51. Yan CH, Faraji F, Prajapati DP, Boone CE, DeConde AS. Association of chemosensory dysfunction and COVID-19 in patients presenting with influenza-like symptoms. Int Forum Allergy Rhinol. 2020; 10(7):806813. doi: $10.1002 /$ alr.22579

52. Bagheri SH, Asghari A, Farhadi M, Shamshiri AR, Kabir A, Kamrava SK, et al. Coincidence of COVID-19 epidemic and olfactory dysfunction outbreak in Iran. Med J Islam Repub Iran. 2020; doi: 10.34171/mjiri.34.62
53. Redondo-Urda MJ, Rodríguez-Peguero FJ, Pérez-Gil O, Del Valle-Sánchez M, Carrera-Izquierdo M. SARSCoV-2, nuevo agente causal del síndrome de GuillainBarré [SARS-CoV-2, a new causal agent of GuillainBarre syndrome]. Rev Neurol. 2020; 71(7):275-276. doi: 10.33588/rn.7107.2020264

54. Virani A, Rabold E, Hanson T, Haag A, Elrufay R, Cheema $\mathrm{T}$, et al. Guillain-Barré Syndrome associated with SARS-CoV-2 infection. ID Cases. 2020; 20:e00771. doi: 10.1016/j.idcr.2020.e00771

55. Sedaghat Z, Karimi N. Guillain Barre syndrome associated with COVID-19 infection: A case report. J Clin Neurosci. 2020 Jun; 76: 233-235. doi: 10.1016/j.jocn.2020.04.062

56. Toscano G, Palmerini F, Ravaglia S, Ruiz L, Invernizzi $\mathrm{P}$, Cuzzoni MG, et al. Guillain-Barré Syndrome Associated with SARS-CoV-2. N Engl J Med. 2020; doi: 10.1056/NEJMc2009191

57.Jin M, Tong Q. Rhabdomyolysis as Potential Late Complication Associated with COVID-19. Emerg Infect Dis. 2020; 26(7):10.3201/eid2607.200445. doi:10.3201/eid2607.200445

58. Gutiérrez-Ortiz C, Méndez A, Rodrigo-Rey S, San Pedro-Murillo E, Bermejo-Guerrero L, Gordo-Mañas R, et al. Miller Fisher Syndrome and polyneuritis cranialis in COVID-19. Neurology. 2020; doi: 10.1212/WNL.0000000000009619

59. Wan Y, Cao S, Fang Q, Wang M, Huang Y. Coronavirus disease 2019 complicated with Bell's palsy: a case report. Research Square. 2020. DOI: 10.21203/rs.3.rs23216/v1

60. Rahman MM, Azam MG, Bohorquez-Rivero J, GarciaBallestas E, Agrawal, Moscote-Salazar LR. Telehealth and telemedicine in the COVID-19 era: a world of opportunities for the neurosurgeons. World Neurosurg. 2020; doi: 10.1016/j.wneu.2020.06.064

61. Lyden P. AHA/ASA Stroke Council Leadership. Temporary emergency guidance to US stroke centers during the COVID-19 pandemic on behalf of the AHA/ASA stroke council leadership. Stroke. 2020; doi: 10.1161/STROKEAHA.120.030023

62. Khosravani H, Rajendram P, Notario L, Chapman MG, Menon BK. Protected Code Stroke: Hyperacute Stroke Management During the Coronavirus Disease 2019 
(COVID-19) Pandemic. Stroke. 2020; doi: 10.1161/STROKEAHA.120.029838 